\title{
Rupture of the Thoracic Duct as a Result of Injury with Subsequent Development of Bilateral Chylothorax
}

\author{
Thomas Bohrer $^{1 *}$, Jens Christian Paul ${ }^{1}$, Konrad Neukam ${ }^{1}$, Oscar Hernandez ${ }^{2}$, Christian O. Ritter ${ }^{3}$, \\ Rudolf Hagen $^{4}$ \\ ${ }^{1}$ The Center of Operative Medicine, Department of Cardiothoracic Surgery, Wuerzburg University, Wuerzburg, Germany; ${ }^{2}$ Hospiten \\ Riviera Maya, Playa del Carmen, Mexico; ${ }^{3}$ Radiology Institute, Wuerzburg University, Wuerzburg, Germany; ${ }^{4}$ Clinic and Policlinic \\ for ENT-Diseases, Wuerzburg University, Wuerzburg, Germany. \\ Email: *thomas.bohrer@sozialstiftung-bamberg.de
}

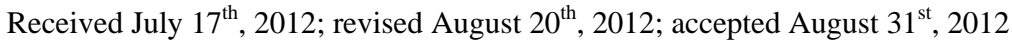

\begin{abstract}
We present a unique case of a 49-year-old woman presenting with a bilateral chylothorax sustained after minor injury. This condition developed secondarily to minor neck injury which resulted in left side cervical swelling. First treatment was conservative. Surgical intervention was nevertheless necessary to remove a cervical cyst contiguous with the thoracic duct.
\end{abstract}

Keywords: Chylothorax; Rupture of Thoracic Duct

\section{Introduction}

Rupture of the thoracic duct is rarely observed. Most often it is accompanied by bilateral cervical swelling. In this case report, we present a bilateral chylothorax sustained after minor injury to the upper thorax.

\section{Case Report}

The patient agreed to publication on grounds of anonymity. The 49-year-old female patient went on a holiday trip to Mexico in 2009. During a guided tour through an underground cave, the patient stumbled, fell and experienced a hyperextension trauma to the cervical spine. Initially the patient reported cervical pain and a decrease of motility of the neck mostly on the left side. After an interval of eight days, the patient reported a sudden increase of pain localized near the left supraclavicular fossa as well as renewed cervical swelling. The patient started to show a large area of swelling in the supraclavicular fossa (Figure 1) and also reported localized pain in the upper half of the collar bone. On the next day swelling started to decrease; however, the patient reported an increase in pain and dyspnoea. Two days later, the cervical swelling completely subsided. Yet, the respiratory function of the patient worsened, resulting in the development of orthopnoea requiring immediate hospitalization.

Upon hospitalization the patient received a chest X-ray

*Corresponding author. showing bilateral pleural effusions. This diagnosis was verified by a subsequent computed tomography of the chest (Figure 2). Consequently the left hemithorax was drained, resulting in the release of $220 \mathrm{~mL}$ of milkywhite secretion. Laboratory analysis showed a massive increase in triglycerides up to $1085 \mathrm{U} / \mathrm{L}$ while glucose and albumin levels were within normal limits resulting in the diagnosis of a chylothorax. Forthwith the patient was put on a fastening regimen for eight days, resulting in successive reduction of the pleural effusions. After this initial therapy, the patient was able to relocate back to Germany, where she presented to the hospital for further

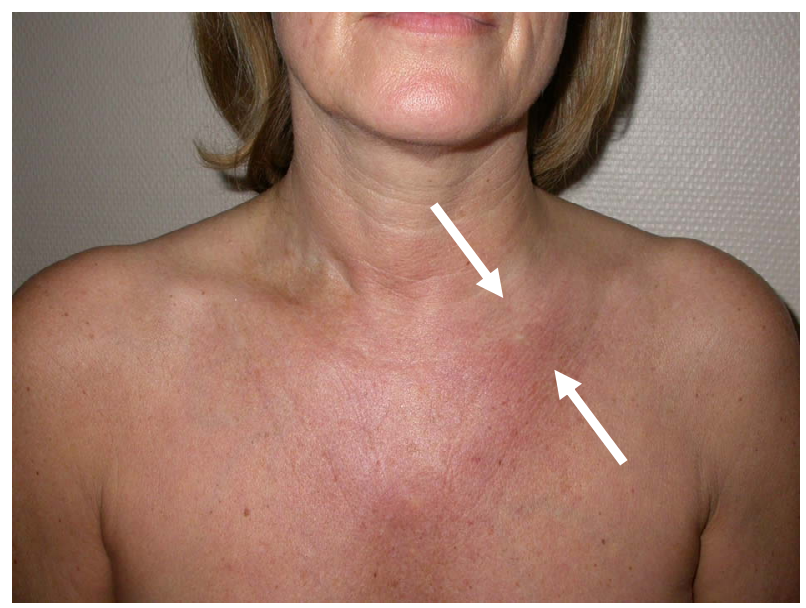

Figure 1. Swelling of the left clavicular fossa several days after minor hyperextension trauma of the cervical spine. 


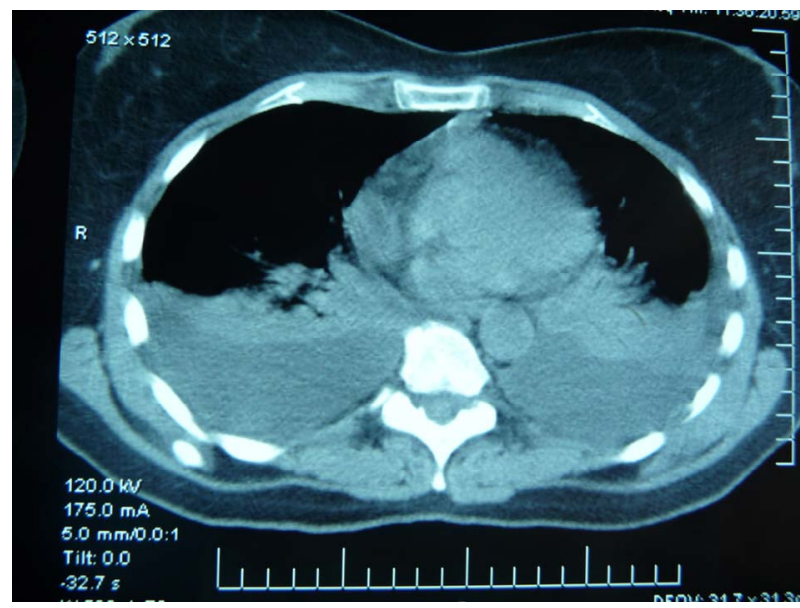

Figure 2. CT scan confirms the pleural effusions seen on prior chest $\mathrm{X}$-ray and excludes concomitant injury as well as hemothorax (Hospiten Riviera Maya, Mexiko).

treatment. Upon hospitalization, an ultrasound scan failed to show a pleural effusion. However, a small $11 \times 9 \times 13$ $\mathrm{mm}$ remnant cystic lesion in the retroclavicular region lateral to the internal jugular vein was visualized (Figure 3). Doppler analysis of the cephalic veins remained unremarkable. An additional MRI of the cervical and thoracic region as well as the mediastinum was inconspicuous as well. Conventional lymphangiography showed an intact thoracic duct without signs of leakage, however the CT scan directly after lymphography a small zystoidic structure next to the thoracic duct most likely corresponding to the cyst remnants after rupture (Figure 4).

Complementary laboratory and blood analysis for infectious disease were negative. Consequently, the patient was discharged from the hospital.

Two months later the patient presented with a recurrent chylothorax and swelling in the left supraclavicular fossa. No physical stress or new trauma had occurred since then. During clinical examination, a new palpable mass was detected, similar to Figure 1. Conclusively, the thoracic cyst located in the left retroclavicular region lateral to the internal jugular vein contiguous with the thoracic duct was surgically removed under general anesthesia by a small supraclavicular incision. Intraoperatively this spot confirmed the leakage point of the chyle. The patient recovered quickly from surgery. Pathology analysis of the resected cyst showed no signs of cestodes or filarial larvae. The patient has since been relapse-free, follow up chest $\mathrm{X}$-rays until now were inconspicuous.

\section{Discussion}

There are multiple etiologies of chylothorax including gunshot wounds, trauma, and birth complications, as well as iatrogenic causes resulting from head and neck, visceral, or thoracic surgery [1]. Congenital defects can also

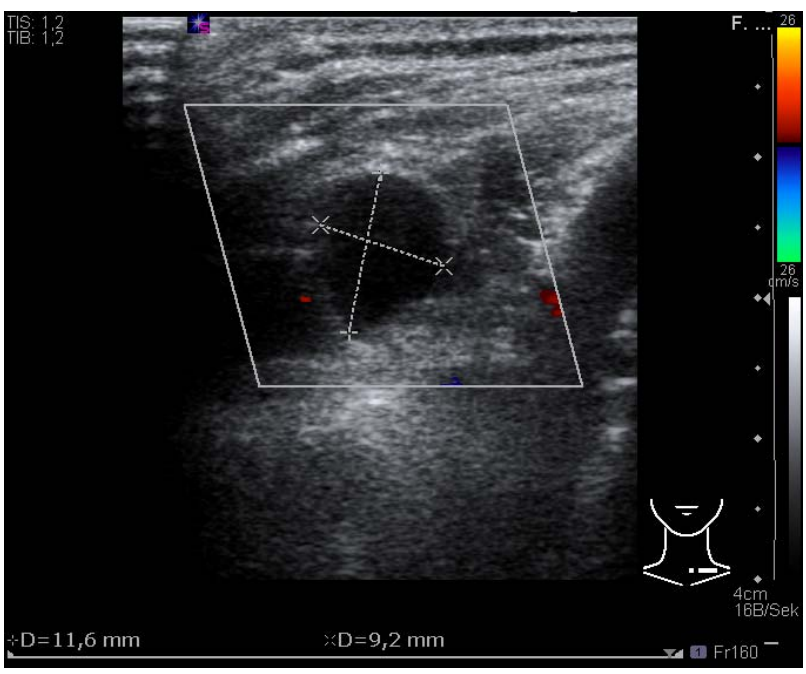

Figure 3. Ultrasound demonstrates a cystic lesion in the left supraclavicular fossa.

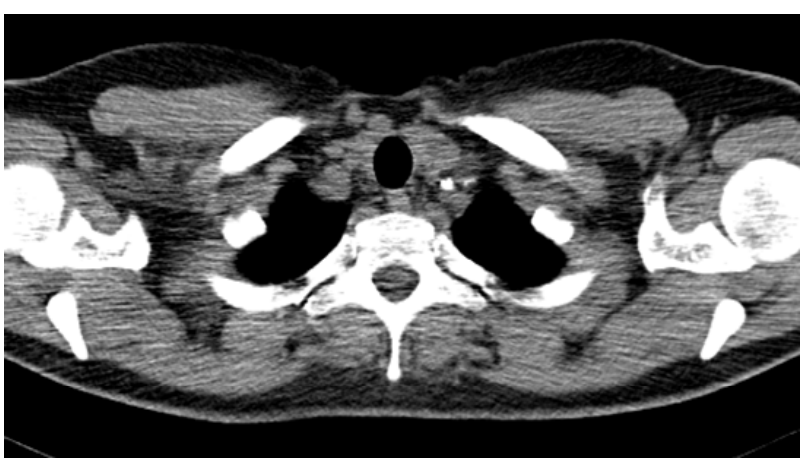

Figure 4. CT scan after lymphography: next to the thoracic duct a small cystic structure is seen caused by Lipiodol contrast most likely equivalent to the cyst remnants after rupture.

result in rupture of the thoracic duct. Ruptured cysts of the duct remain a rarity; literature review shows only a few isolated cases [2]. However, a rupture with consecutive development of a bilateral chylothorax has not been described so far in the international literature, the mechanism of its development is still unclear. Besides taking a thorough patient history, clinical examination and pleural drainage, a wide range of diagnostic tests can be utilized including MRI (which in our case showed no findings). Sometimes, milk administration before surgery can help to find the leakage point of the chyle when not clearly detected as in or case by clinical examination, ultrasound or CT scan. Even in the circumstances of minor trauma such as in this case, an infectious, parasitic [3], or neoplastic (e.g. lymphoma or leukaemia [4]) cause should be considered and tested. It is also important to perform a conventional lymphangiography, an established but yet nearly forgotten diagnostic procedure, to allow for proper diagnosis of regional spread [5]. 


\section{Acknowledgements}

There is no conflict of interest or financial support of the project, grant, technical or other assistance concerning this paper.

\section{REFERENCES}

[1] F. Benedix, H. Lippert and F. Meyer, "Post-Surgical Lymphocutaneous Fistula, Chylous Ascites and Chylothorax-Infrequent but Serious Complications: Etiology, Diagnosis and Therapeutic Options,” Zentralblatt für Chirurgie, Vol. 132, No. 6, 2007, pp. 529-538. doi:10.1055/s-2007-981364

[2] B. Balestra, "An Unusual Recurrent Neck Swelling,"
Schweizer Medizinische Wochenschrift, Vol. 127, No. 1457, 1997, p. 1457.

[3] T. Matsumoto, T. Yamagami, T. Kato, et al., "The Effectiveness of Lymphography as a Treatment Method for Various Chyle Leakages,” British Journal for Radiology, Vol. 82, No. 976, 2009, pp. 286-290. doi:10.1259/bjr/64849421

[4] H. Wessder, "Malignant Lymphoma,” In: M. J. Viamonte and A. Rüttimann, Eds., Atlas of Lymphography, ThiemeStratton, Stuttgart, 1980.

[5] A. Guermazi, P. Brice, C. Hennequin and E. Sarfati, "Lymphography: An Old Technique Retains Its Usefulness,” Radiographics, Vol. 23, No. 11, 2003, pp. 15411558. doi:10.1148/rg.236035704 